\title{
Development of Syndrome Definitions for Acute Unintentional Drug and Heroin Overdose
}

\author{
Em Stephens* \\ Virginia Department of Health, Richmond, VA, USA
}

\section{Objective}

To develop and evaluate syndrome definitions for the identification of acute unintentional drug overdose events including opioid, heroin, and unspecified substances among emergency department (ED) visits in Virginia.

\section{Introduction}

Nationally, deaths due to opioid overdose have continually increased for the past 15 years $^{1}$. Deaths specifically related to heroin increased more than four-fold between 2002 and 20142. Hospital inpatient discharge data provide information on non-fatal overdoses, but include a significant lag in reporting time ${ }^{3}$. Syndromic ED visit data provide near real-time identification of public health issues and can be leveraged to inform public health actions on the emerging threat of drug overdose.

\section{Methods}

Virginia Department of Health (VDH) developed two syndrome definitions in 2014 to capture acute unintentional drug overdose events among syndromic ED visit data. Syndrome 1 captured visits for overdose, whether or not a specific substance was mentioned. Syndrome 2 captured only visits for heroin overdose. Definitions were based on free-text terms found within the chief complaint and standardized text or International Classification of Diseases (ICD) codes within the diagnosis field. In 2016, both definitions were revised to identify additional inclusion and exclusion criteria according to CDC guidance documentation and syndrome definitions used by other state jurisdictions.

Microsoft SQL was used to modify both definitions based on the newly identified chief complaint and diagnosis criteria. Record level data were analyzed for their adherence to established criteria using an iterative evaluation process.

The scope of Syndrome 1 (2016) was narrowed from the 2014 version by excluding visits for non-opioid substances, heroin, and non-acute indicators. It included chief complaint and diagnosis terms related to opioids, unspecified substance overdose, narcotics, and Narcan or naloxone, and excluded terms related to suicide, alcohol overdose alone, withdrawal, detoxification, rehab, addiction, constipation, chronic pain, and any specified non-opioid drug or medication. Syndrome 2 (2016) included chief complaint or diagnosis terms mentioning heroin overdose and excluded suicide, withdrawal, detoxification, rehab, and addiction. Visits with mention of suicide, rehab, or addiction were identified during the evaluation process, resulting in the exclusion of these terms in the revised query.

From January 1, 2015 to July 31, 2016, the number of visits captured by the revised syndrome definitions was compared to the number captured by the 2014 definitions. Correlation coefficients were calculated using SAS 9.3.

\section{Results}

The revised Syndrome 1 found 4296 fewer ED visits (29\% decrease) for acute unintentional drug overdose between January 1, 2015 and July 31, 2016 compared to the 2014 definition. Despite the drop in volume, the monthly trends were similar for the 2014 and 2016 definitions (correlation coefficient $=0.95$, $\mathrm{p}<0.001)$. For the same time period, the revised Syndrome 2 definition returned 108 fewer visits ( $6 \%$ decrease) for acute unintentional heroin overdose. The monthly trends were also similar for the 2014 and 2016 definitions (correlation coefficient $=0.98, \mathrm{p}<0.001$ ).

\section{Conclusions}

Both revised syndrome definitions improved specificity in capturing overdose visits as Syndrome 1 (2016) identified 29\% fewer visits and Syndrome 2 (2016) identified 6\% fewer visits found to be unrelated to the desired overdose criteria.

When developing the revised syndrome definitions, VDH decided to exclude non-acute drug-related visits. Terms such as addiction, detoxification, rehab, withdrawal, chronic pain, and constipation were indicative of habitual drug use or abuse instead of acute overdose and were thus excluded. In narrowing the scope of Syndrome 1, VDH also identified and excluded visits for specified drug and medication overdose. Together, these expanded exclusion criteria resulted in greater specificity with both updated syndromes.

These revised syndrome definitions enable VDH to better track opioid and heroin overdose trends in near real-time and over extended time periods which can be used to inform public health actions. Limitations include the inconsistency of diagnosis coding among syndromic data submitters, which may lead to geographic underrepresentation of unintentional drug overdose visits based on the location of health care systems. VDH will continue to evaluate and refine these overdose syndrome definitions as this emerging health issue evolves.

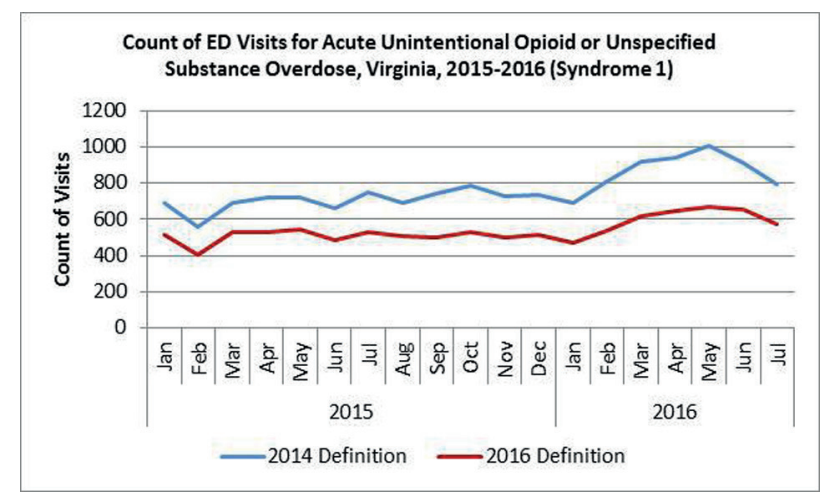


ISDS 2016 Conference Abstracts

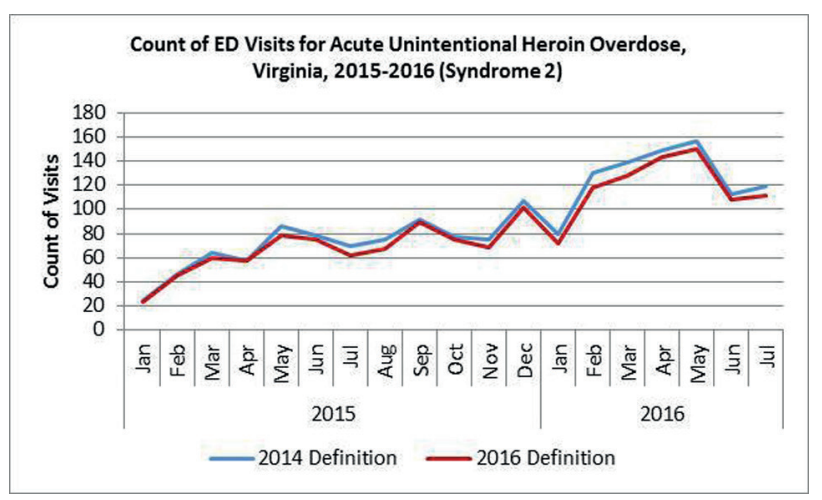

\section{Keywords}

Drug Overdose; Opioids; Syndrome Definition; Syndromic Surveillance

\section{Acknowledgments}

Erin E. Austin

\section{References}

1. CDC.org [Internet]. Atlanta: Injury Prevention and Control: Opioid Overdose; [updated 2016 Mar 14; cited 2016 Sept 2]. Available from: https://www.cdc.gov/drugoverdose/opioids/heroin.html

2. CDC.org [Internet]. Atlanta: Wide-Ranging Online Data for Epidemiologic Research (WONDER); [updated 2016 Jul 12; cited 2016 Sept 2]. Available from: http://wonder.cdc.gov

3. CDC.org [Internet]. Atlanta: Injury Prevention and Control: Data \&Statistics (WISQARS); [updated 2016 May 10; cited 2016 Sept 2]. Available from: http://www.cdc.gov/injury/wisqars/facts.html.

\section{${ }^{*}$ Em Stephens}

E-mail: emily.stephens@vdh.virginia.gov 